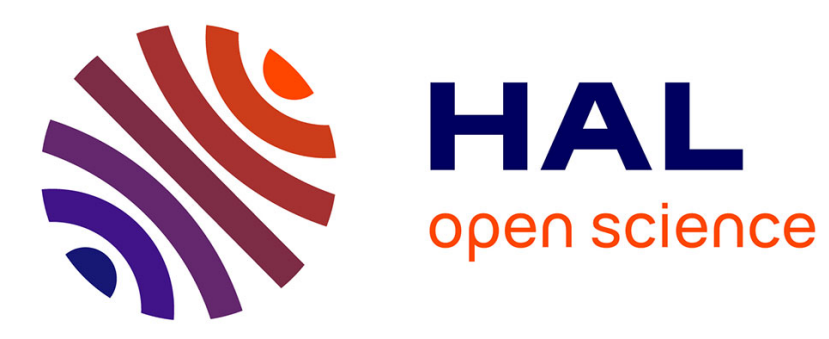

\title{
Biology of multiciliated cells
}

\author{
Camille Boutin, Laurent Kodjabachian
}

\section{To cite this version:}

Camille Boutin, Laurent Kodjabachian. Biology of multiciliated cells. Current Opinion in Genetics and Development, 2019, 56, pp.1-7. 10.1016/j.gde.2019.04.006 . hal-02347294

\section{HAL Id: hal-02347294 \\ https://hal.science/hal-02347294}

Submitted on 5 Nov 2019

HAL is a multi-disciplinary open access archive for the deposit and dissemination of scientific research documents, whether they are published or not. The documents may come from teaching and research institutions in France or abroad, or from public or private research centers.
L'archive ouverte pluridisciplinaire HAL, est destinée au dépôt et à la diffusion de documents scientifiques de niveau recherche, publiés ou non, émanant des établissements d'enseignement et de recherche français ou étrangers, des laboratoires publics ou privés. 


\section{BIOLOGY OF MULTICILIATED CELLS}

Camille Boutin and Laurent Kodjabachian*

Aix Marseille Univ, CNRS, IBDM, Marseille, France

*correspondence to : laurent.kodjabachian@univ-amu.fr

Keywords: Multiciliated cells, centriole, cilia, deuterosome, polarity 


\section{Abstract}

Multiciliated cells (MCCs) are specialized in fluid propulsion through directional beating of myriads of superficial motile cilia, which rest on modified centrioles named basal bodies. MCCs are found throughout metazoans, and serve functions as diverse as feeding and locomotion in marine organisms, as well as mucus clearance, cerebrospinal fluid circulation, and egg transportation in mammals. Impaired MCC differentiation or activity causes diseases characterized by severe chronic airway infections and reduced fertility. Through studies in Xenopus and mouse mainly, MCC biology has made significant progress on several fronts in recent years. The gene regulatory network that controls MCC specification and differentiation has been deciphered to a large extent. The enigmatic deuterosomes, which serve as centriole amplification platforms in vertebrate MCCs, have started to be studied at the molecular level. Principles of ciliary beating coordination within and between MCCs have been identified.

\section{Introduction}

Multiciliated cells are defined by the presence at their surface of few dozens to hundreds of cilia, which beat coordinately to generate robust polarized hydrodynamic forces (Fig. 1). Each cilium is templated by a basal body (BB), a modified centriole, which harbors two asymmetric appendages, a rootlet that plunges into the cytoplasm, and a basal foot that points in the beating direction (Fig. 1A). Through their interaction with cytoskeletal elements, these appendages link BBs together, thus coordinating cilia orientation [1]. MCCs can be found as individual cells (e.g. cycad plant male gametes or Paramecium) or organized into specialized epithelia in metazoans [2]. In small-sized aquatic invertebrate organisms, MCCs are mainly involved in locomotion, whereas in vertebrates, MCCs help propel biological fluids at the surface of specialized tissues. Three main models have helped advancing our knowledge of 
fundamental principles of MCC biology, the ciliated epidermis of the Xenopus embryo (Fig. 1BE), the airway mucociliary and the brain ependymal (Fig. $1 \mathrm{~B}^{\prime}-\mathrm{E}^{\prime}$ ) epithelia of the mouse. Production of efficient directional fluid flows depends on several parameters integrated in time and space during development, as well as during regeneration. First, a correct number of MCCs must be produced, second a correct number of functional cilia must be assembled in each MCC, third cilia must coordinate their beating orientation within individual and between neighboring MCCs to produce robust and regular strokes. In humans, disruption of cilia production or beating in MCCs leads to hydrocephalus, respiratory and fertility pathologies [1]. In this review, we shall focus on recent findings regarding the transcriptional control of MCC differentiation, the mechanisms of centriole synthesis, and the multiscale organization of polarized ciliary beating.

\section{Establishment and maintenance of MCC identity}

In all models, MCC fate adoption appears to be triggered by Notch pathway inhibition, which launches a specific gene regulatory network (GRN), whose key nodes are named Gemc1 (Gmnc), Mcidas (Multicilin), Myb, FoxJ1 and Rfx2/3 [1] (Fig. 2). BMP pathway inhibition can also turn on the MCC GRN in Xenopus and in cultures of human airway epithelium [3]. Gemc1 and Mcidas belong to the Geminin family of nuclear factors, and together with E2F family transcription factors are necessary and sufficient to trigger multiciliogenesis $[1,4]$. Both Gemc1 and Mcidas can activate Myb, which promotes centriole multiplication, as well as FoxJ1 and $\operatorname{Rf} \times 2 / 3$, which coordinately control motile ciliogenesis $[1,5]$. A recent study revealed that in mouse, MCC fate is controlled by Gemc1, while Mcidas acts downstream to initiate centriole amplification, providing a rationale for the sequential involvement of two related genes that until now appeared to share the same activity [6]. The core MCC GRN has recently been 
enriched with additional regulators that branch at distinct points (Fig. 2). Among those, the chromatin factor Trrap appears to function downstream of Notch and upstream of Mcidas in human airway MCCs [7]. Trrap was identified through a pool-based shRNA screen, demonstrating the value of unbiased phenotypic searches to identify additional MCC regulators. The p53 family member, p73, was shown to regulate about 100 ciliary genes, including FoxJ1 and $\mathrm{Rf} \times 2 / 3$, and p73 mutant mouse display typical features linked to MCC dysgenesis such as hydrocephalus, sterility and chronic airway inflammation/infection $[8,9]$. Interestingly, it was suggested that p73 marks some basal cells in airway epithelium for MCC differentiation, lending to it a role as a pioneer or competence factor [8]. Further supporting the importance of $\mathrm{p73}$, it was also reported that it regulates ependymal MCC polarity through maintenance of actin and microtubule cytoskeletal networks [10]. It is important to mention that the MCC identity is inherently labile, as its maintenance requires constant FoxJ1 transcriptional activity, at least in murine ependymal MCCs [11].

\section{Centriole synthesis in MCCs}

A key step of MCC differentiation is the large-scale production of centrioles that will subsequently be converted into BBs to template motile cilia. In vertebrate MCCs, it is achieved via two pathways: the parental centriole-dependent pathway, which is analogous to the duplication pathway active during cell cycle, but can produce 10-20 centrioles in MCCs; the deuterosome-dependent pathway, which produces $80-90 \%$ of total MCC centrioles [1,12] (Fig. 3). Deuterosomes comprise non-centriolar electron-dense structures that can support the growth of single or multiple procentrioles according to their size, which may increase over differentiation time [13-15]. Despite its initial description in the late 70's, the deuterosome remained virtually unexplored for over 40 years. Recent molecular studies started to unveil 
the composition of Xenopus and mouse deuterosomes [13-16]. These studies have shown that the architecture of this organelle is based on a set of scaffolding proteins including Deup1 at its core, Pericentrin, $\gamma$-tubulin and Cep152 at its periphery. The three latter proteins are wellknown components of the pericentriolar material (PCM) [17], which underlines an unexpected link between deuterosome and PCM. Refined mapping of Deup1, Pericentrin and $\gamma$-tubulin revealed the existence of nested subdomains in deuterosomes that require further investigation [14]. For instance, it will be important to understand how these domains are assembled and evolve over deuterosome lifetime. In addition, the functional relevance of such organization for deuterosome function remains to be addressed.

Deciphering the sub-cellular origin of the deuterosome may provide interesting cues on how it is built. Questioning the long-held belief that centriole synthesis by deuterosomes occurs de novo (i.e. independently from centrosomal centrioles), live imaging of newly synthesized centrioles suggested that in cultured mouse ependymal cells, deuterosomes are seeded by the daughter centrosomal centriole [15]. However, using both chemical and genetic approaches, three independent studies now suggest that formation of deuterosomes and production of multiple centrioles can occur in absence of parental centrioles (Zhao et al., bioRxiv doi.org/10.1101/373662; Nanjundappa et al., bioRxiv doi.org/10.1101/478297; Mercey et al., bioRxiv doi.org/10.1101/503730). Thus, it is unclear whether deuterosomes are seeded by or transit through parental centrioles, which can only be resolved by live imaging of deuterosomes and centrioles in parallel. Seeking for the mechanisms of deuterosome construction, it is interesting to remember that this organelle is the second entity observed during mass centriole production, the first one being fibrous granules (FGs). Apart from early EM studies that described them as electron dense granules that condensate to form deuterosomes, FGs remain marginally characterized [12]. A notable exception is the 
identification of PCM1, a specific marker labelling FGs [18], but for which no role in multiciliogenesis could be detected [19]. However, a recent study showed that: i. Deup1 and PCM1 proteins co-localize during the initial steps of MCC differentiation; ii. after MCC transcriptional program is launched, E2F4, a co-activator of Multicilin, shuttles from the nucleus to the cytoplasm where it localizes in the PCM1 positive cloud of material and participates to deuterosome formation [20]. Therefore, further (re-)evaluating the role of FGs during MCC differentiation is likely to provide information about the construction and/or function of the deuterosome.

Imaging centriole and deuterosome markers in real-time and on fixed samples revealed three successive phases: templating of procentrioles, growth of procentrioles and disengagement of mature centrioles from deuterosomes and parental centrioles [13-15,21] (Fig. 3). Molecular regulators and effectors operating in those phases have been identified. A set of major cell cycle regulators including CDK2, CDK1, PLK1 and APC/C control transitions between the different phases [21,22]. Highlighting more detailed mechanistic aspects of the disengagement phase, a recent study reported the existence of a proteolytic cascade involving the Separase protease and CDC20B to release centrioles from deuterosomes [14]. These complementary findings opened large avenues to understand deuterosome-mediated centriole production. For instance, the implication of cell cycle kinases calls for the identification of their targets in MCCs. Furthermore, it would be interesting to determine whether - similar to what happens during the cell cycle [23] - those kinases are counteracted by specific phosphatase activities.

\section{MCC polarized beating, a multi-step and multiscale problem}


Multiciliated epithelia are specialized to ensure the vectorial transport of particles or cells along their surface. Their effective function relies on the coordination of ciliary beating within individual cells and at the tissue level. This is achieved through a multistep process involving events occurring both at the sub-micrometer scale and at the scale of the entire organ or organism.

Centrioles newly released in the cytoplasm are transported towards the apical surface. Meanwhile, they are converted into BBs by the addition of rootlet and basal foot appendages [1] (Fig. 3). The precise mechanisms of BB maturation and transport remain poorly understood but seem to implicate acto-myosin and the formation of vesicles at the distal appendages of BBs $[24,25]$.

Once they arrive to their destination, BBs organize at the apical surface. Their distribution varies between different types of MCCs. In Xenopus epidermis, BBs are evenly dispersed at the surface of the MCC [26] (Fig. 1E). In contrast, BBs are organized in rows covering the entire apical surface in mouse tracheal MCCs [27]. In mouse ependymal MCCs, BBs are clustered in an off-centered patch, through a process called translational polarity (Fig. 1D'). Within this patch, BBs are organized in rows $[28,29]$ (Fig. 1E'). Whether such variations in BB organization underlie functional differences remains to be determined. In all types of MCCs, BBs of a given cell are oriented in the same direction. This peculiar organization, referred to as rotational polarity, is essential to ensure the coordinated beating of cilia within individual MCCs.

Both distribution and orientation of BBs relies on their tight interaction with apical cytoskeletal elements that differentially contribute to the geometry of the array. Cortical actin in MCCs is organized in two structurally distinct apical and sub-apical networks $[26,27,30,31]$. The apical pool is necessary for docking of BBs to the cell membrane $[24,26]$. The sub-apical network connects BBs and is necessary for their correct spacing [26,32]. Microtubules 
connecting BBs play an instrumental role in their alignment and orientation $[26,27]$. Finally, a network of intermediate filaments is also observed unsheathing BBs, although its precise functional contribution remains to be determined $[27,31]$.

Interplay between those cytoskeletal elements and their interacting proteins results in the BB organization described above [33-37]. Among those, some unexpected proteins were identified. For instance, FAK, Paxillin and Vinculin - well-known focal adhesion proteins associate with BBs and their striated rootlet to form ciliary adhesion complexes that connect BBs to the actin cytoskeleton [34,35]. Likewise, the well-characterized chromatin modifier WDR5 acts as a scaffolding protein binding to BBs and modulating apical actin in MCCs [37]. Beyond its role in BB organization, the apical cytoskeleton of MCCs is thought to be important for propagation of the metachronal wave of ciliary beating [26]. It also confers a mechanical resistance to BBs to sustain the shear stress imposed by ciliary beating in mature MCCs $[30,38]$. Ciliary activity itself provides an important feedback on BB organization. Although initially imperfectly coordinated, cilia beating instructs weak directional flow that improves coordination of BB polarity, which in turn reinforces the flow $[39,40]$. However, the precise range of action of such hydrodynamic feedback remains to be evaluated. In addition, cilia motility is necessary for apical actin meshwork assembly, thereby reinforcing BB anchoring at the apical surface [30].

To generate a directional fluid flow and sustain the correct function of the organ, ciliary beating must be coordinated at the tissue-level. This is achieved through a planar cell polarity driven multistep process $[29,41-44]$. The initial cue that establishes organ-level polarity remains unknown in mouse ciliated epithelia. In contrast, it was shown that the mechanical strain transmitted from the mesoderm to the ectoderm during Xenopus gastrulation caused alignment of microtubule arrays along the rostro-caudal axis, which secondarily led to PCP 
protein asymmetric distribution along the same axis. Through this global polarization of the epithelium, individual MCCs acquire polarized membrane identity. This generates a compass in each MCC that is used to orient the BB array, thereby allowing cilia beating direction to align with global tissue polarity $[29,43,45]$. Unexpectedly, it was reported that maintenance of tissue-level polarity in airways requires the presence of a sufficient number of MCCs for cellcell transmission of PCP polarization [46]. Consequently, diseases that cause loss of MCCs, such as cystic fibrosis, are characterized by poor tissue polarity, which may worsen mucociliary clearance [46].

While tissue- and cell-level scales of organization start to be well described [41], submicrometer scale events remains to be understood. For instance, structural and molecular chirality of BBs need to be resolved at very high resolution to understand their function (N'Guyen et al., biorXiV doi.org/10.1101/487330). Since BB structural organization varies between different types of MCCs, such studies will also help to understand functional differences between MCCs. At the opposite end of the spectrum, MCC activity needs to be integrated at the millimeter-scale to generate effective flows. Here, mechanical interactions between cilia and the fluid they propel become highly significant [47]. To fully understand ciliated epithelium biology, future studies should aim at bridging sub-micrometer to millimeter scales, and at integrating molecular, structural and mechanical dimensions.

\section{Conclusions}

The field of MCC biology has advanced at fast pace in recent years. However, beyond the findings described above and the questions they open, many issues remain to be addressed. 
A first problem regards the degree of universality of the principles identified so far. For instance, it is unclear whether MCCs in invertebrates use deuterosome-like structures to amplify their centrioles. In opposition to this idea, Mcidas and Deup1 genes appear to be absent in invertebrate genomes. This issue may receive light from planarians, which lack centrosomes, but make full-fledged epidermal MCCs required for locomotion [48](Thi-Kim et al., bioRxiv doi.org/10.1101/324822). On a different note, the various steps driving centriole biogenesis were reported to be highly synchronous in individual cultured mouse ependymal MCCs $[15,21]$. It is unclear whether such synchrony applies to all types of MCCs, or reflects the peculiar organization of ependymal cilia into tufts of relatively constant size.

In future, it will be important to address the architecture and the biophysical nature of the deuterosome. Compartmentalization is used by cells to concentrate biochemical reactions. In most organelles, biochemical reactions are constrained by a membrane. Alternatively, organelles that lack delimiting membrane such as the PCM are formed through complex phase-separation mechanisms to create small volumes for specific biochemical reactions in the cytoplasm [49]. Such liquid-like organelles have recently been reported in MCCs to serve as platforms of assembly of axonemal dynein arms to ensure ciliary beating [50]. It is tempting to speculate that based on its shared features with the PCM, the deuterosome may also comprise a phase-separated organelle, which would concentrate elementary parts and enzymes to build up multiple centrioles.

An overarching question in the field concerns the control of the final number of centrioles present in individual MCCs. Although this number can be highly variable in Xenopus epidermal MCCs or mouse tracheal MCCs, and less so in mouse ependymal MCCs, it always scales to the apical area of the cell (unpublished results; Nanjundappa et al., bioRxiv doi.org/10.1101/478297). Understanding such scaling represents a challenging but fascinating 
question, with fundamental as well as biomedical implications, for instance to better fight cancer cells, which are often characterized by excess number of centrioles.

It has become clear that centriole synthesis during the cell cycle and in MCCs share many molecular and regulatory principles. This leads to the idea that centriole duplication on the one hand and centriole mass production on the other hand are indeed extreme cases of one and the same biological process: centriole biogenesis. The question then becomes: what variations shape those pathways such that only one or hundreds of centrioles are produced, and can such variations account for centriole over-duplication in cancer?

\section{Acknowledgements}

We apologize to authors whose work could not be quoted due to space constraints. Work in LK's laboratory is supported by grants from Agence Nationale de la Recherche, Fondation pour la Recherche Médicale, Fondation ARC, and Ligue Nationale contre le cancer. 

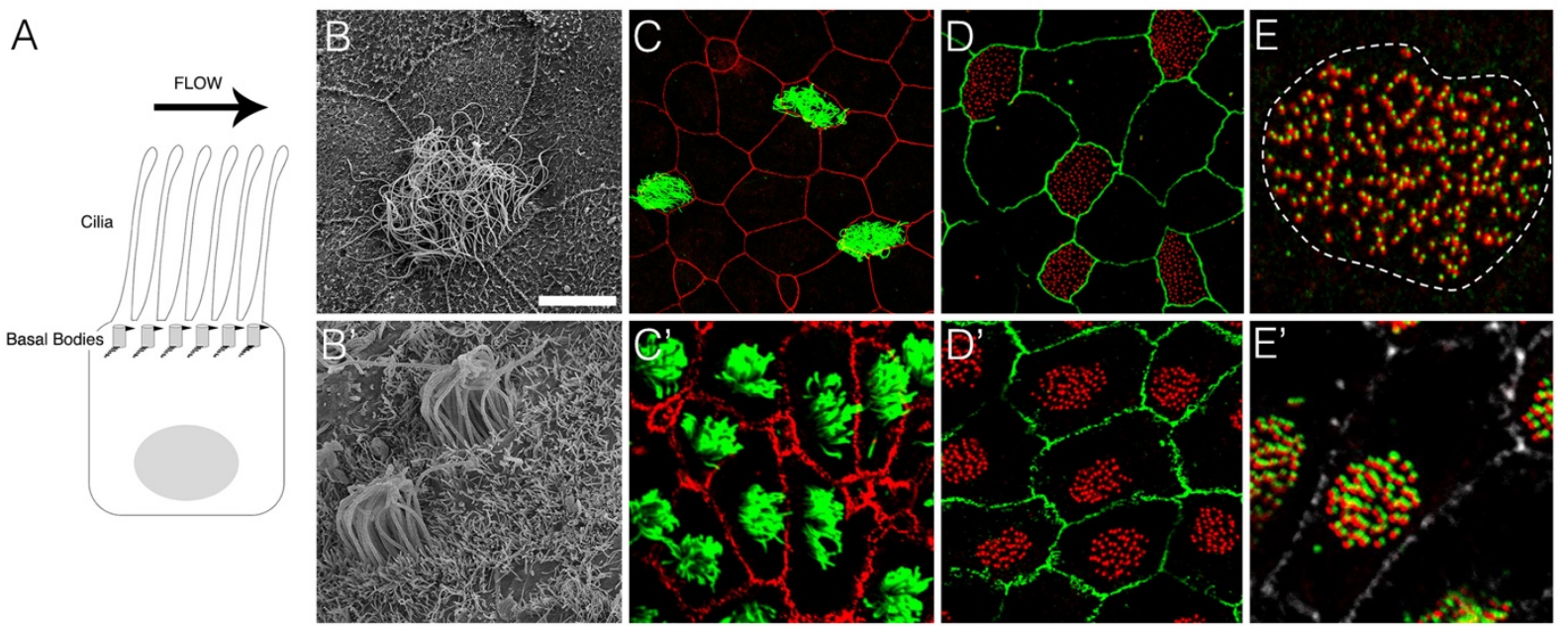

Figure 1: Multiciliated cells in Xenopus epidermis and mouse ependyma

(A) Scheme depicting MCC organization. (B, B') Scanning Electron Microscope micrographs of MCCs from Xenopus epidermis (B) and mouse ependyma ( $\left.B^{\prime}\right)$. (C-E') Confocal pictures of fluorescent staining on Xenopus epidermis (C-E) and mouse ependyma $\left(C^{\prime}-E^{\prime}\right) .\left(C, C^{\prime}\right)$ Staining for the junction marker ZO1 (red) and the cilia marker acetylated- $\alpha$-tubulin (green). (D, D') Staining for the junction marker ZO1 (green) and BB markers centrin (D) and FOP (D') (red). (E, $\left.E^{\prime}\right)$ Zoom on individual MCCs stained with centriole marker centrin (E) or FOP (E') and basal foot market $\gamma$-tubulin (red). Scale bar: $10 \mu \mathrm{m}(\mathrm{B}), 25 \mu \mathrm{m}(\mathrm{C}, \mathrm{D}), 5 \mu \mathrm{m}(\mathrm{E}, \mathrm{F}, \mathrm{I}), 2,5 \mu \mathrm{m}(\mathrm{G}, \mathrm{H})$. 


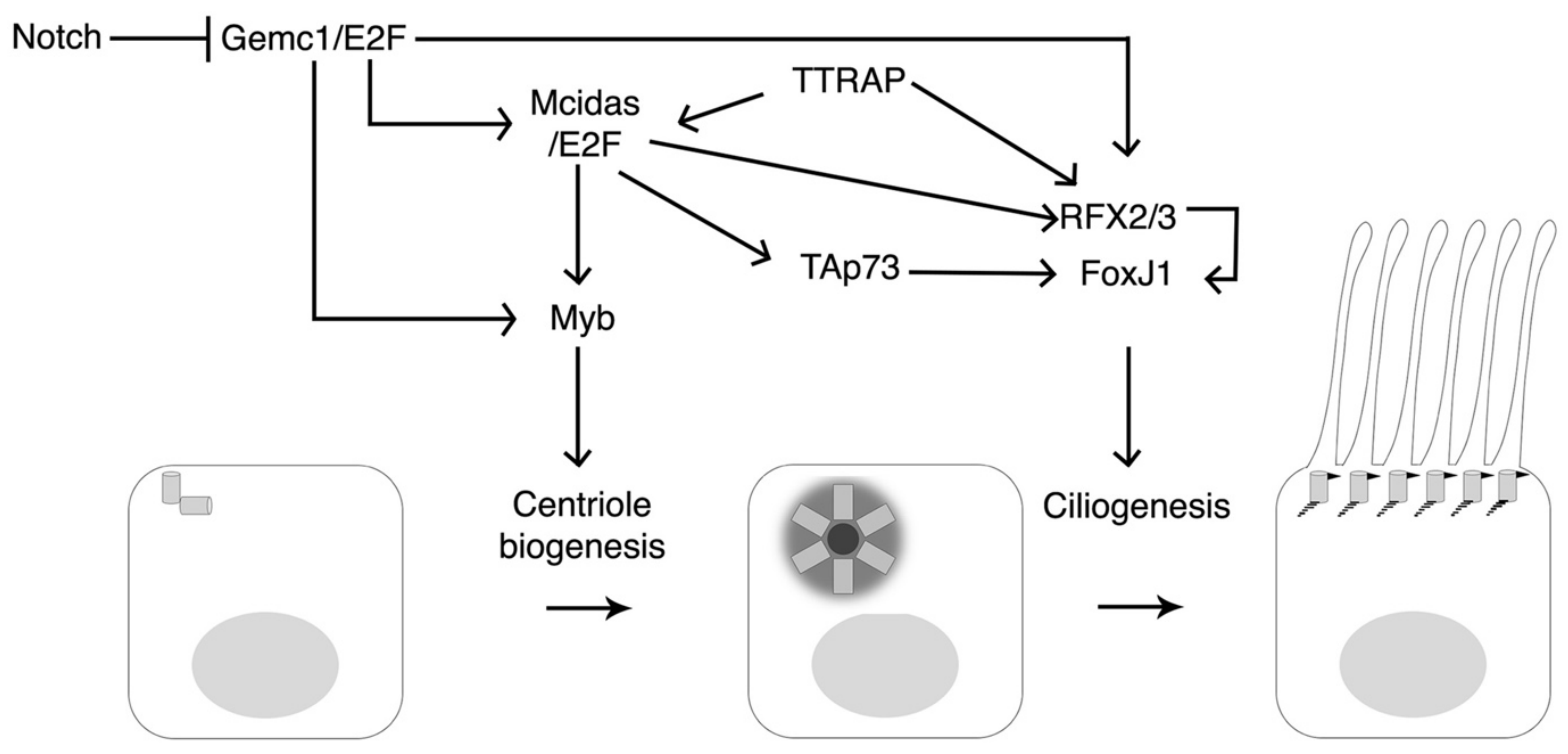

Figure 2: Gene regulatory network at play during MCC differentiation 


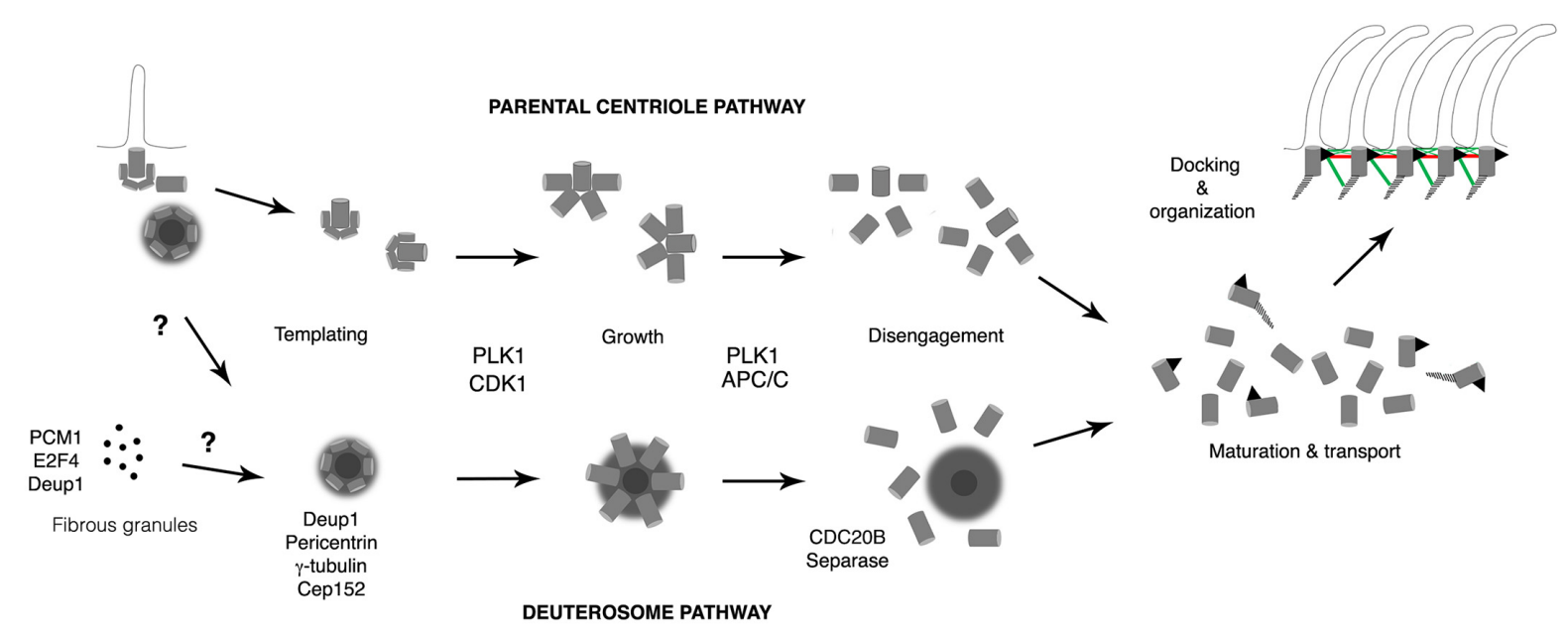

Figure 3: Centriole production and maturation in MCCs

The authors declare no conflict of interest. 


\section{References}

1. Spassky N, Meunier A: The development and functions of multiciliated epithelia. Nat Rev Mol Cell Biol 2017, 18:423-436.

2. Meunier A, Azimzadeh J: Multiciliated Cells in Animals. Cold Spring Harb Perspect Biol 2016, 8.

3. Cibois M, Luxardi G, Chevalier B, Thome V, Mercey O, Zaragosi LE, Barbry P, Pasini A, Marcet $B$, Kodjabachian L: BMP signalling controls the construction of vertebrate mucociliary epithelia. Development 2015, 142:2352-2363.

4. Kim S, Ma L, Shokhirev MN, Quigley I, Kintner C: Multicilin and activated E2f4 induce multiciliated cell differentiation in primary fibroblasts. Sci Rep 2018, 8:12369.

\section{Quigley IK, Kintner C: Rfx2 Stabilizes Foxj1 Binding at Chromatin Loops to Enable} Multiciliated Cell Gene Expression. PLoS Genet 2017, 13:e1006538.

6. Lu H, Anujan $\mathrm{P}$, Zhou F, Zhang $\mathrm{Y}$, Ling Chong $\mathrm{Y}$, Bingle $\mathrm{CD}$, Roy S: Mcidas mutant mice reveal a two-step process for the specification and differentiation of multiciliated cells in mammals. Development 2019, In press.

*7. Wang Z, Plasschaert LW, Aryal S, Renaud NA, Yang Z, Choo-Wing R, Pessotti AD, Kirkpatrick ND, Cochran NR, Carbone W, et al.: TRRAP is a central regulator of human multiciliated cell formation. J Cell Biol 2018, 217:1941-1955.

This paper illustrates how novel essential regulators of MCC biogenesis can be identified via unbiased phenotypic screens.

8. Marshall CB, Mays DJ, Beeler JS, Rosenbluth JM, Boyd KL, Santos Guasch GL, Shaver TM, Tang $\amalg$, Liu $Q$, Shyr $Y$, et al.: p73 Is Required for Multiciliogenesis and Regulates the Foxj1Associated Gene Network. Cell Rep 2016, 14:2289-2300.

9. Nemajerova A, Kramer D, Siller SS, Herr C, Shomroni O, Pena T, Gallinas Suazo C, Glaser K, Wildung $M$, Steffen $\mathrm{H}$, et al.: TAp73 is a central transcriptional regulator of airway multiciliogenesis. Genes Dev 2016, 30:1300-1312.

10. Fuertes-Alvarez S, Maeso-Alonso L, Villoch-Fernandez J, Wildung $M$, Martin-Lopez $M$, Marshall C, Villena-Cortes AJ, Diez-Prieto I, Pietenpol JA, Tissir F, et al.: p73 regulates ependymal planar cell polarity by modulating actin and microtubule cytoskeleton. Cell Death Dis 2018, 9:1183.

*11. Abdi K, Lai CH, Paez-Gonzalez P, Lay M, Pyun J, Kuo CT: Uncovering inherent cellular plasticity of multiciliated ependyma leading to ventricular wall transformation and hydrocephalus. Nat Commun 2018, 9:1655.

This paper highlights the need for constant transcriptional input from FoxJ1 to maintain the MCC phenotype, a principle that is shared by other terminally differentiated cell types. 
12. Nabais C, Pereira SG, Bettencourt-Dias M: Noncanonical Biogenesis of Centrioles and Basal Bodies. Cold Spring Harb Symp Quant Biol 2017, 82:123-135.

\section{Zhao H, Zhu L, Zhu Y, Cao J, Li S, Huang Q, Xu T, Huang X, Yan X, Zhu X: The Cep63} paralogue Deup1 enables massive de novo centriole biogenesis for vertebrate multiciliogenesis. Nat Cell Biol 2013, 15:1434-1444.

**14. Revinski DR, Zaragosi LE, Boutin C, Ruiz-Garcia S, Deprez M, Thome V, Rosnet O, Gay AS, Mercey $O$, Paquet $A$, et al.: CDC20B is required for deuterosome-mediated centriole production in multiciliated cells. Nat Commun 2018, 9:4668.

This paper reports for the first time the specific transcriptomic signature of MCCs at the stage of deuterosome-mediated centriole amplification. It also identifies g-tubulin and PCNT as deuterosome cradle components, thus highlighting an unexpected analogy to the PCM. Using STED super-resolution microscopy, this study helped to better define the architecture of the deuterosome, and put forward the notion of PDM (PeriDeuterosomal Material), which now requires functional analysis. Finally, this work highlighted the adaptation of ancestral and newly evolved cell-cycle related factors to support deuterosome function.

15. Al Jord A, Lemaitre AI, Delgehyr N, Faucourt M, Spassky N, Meunier A: Centriole amplification by mother and daughter centrioles differs in multiciliated cells. Nature 2014, 516:104-107.

16. Klos Dehring DA, Vladar EK, Werner ME, Mitchell JW, Hwang P, Mitchell BJ: Deuterosome-mediated centriole biogenesis. Dev Cell 2013, 27:103-112.

17. Woodruff JB, Wueseke O, Hyman AA: Pericentriolar material structure and dynamics. Philos Trans R Soc Lond B Biol Sci 2014, 369.

18. Kubo A, Sasaki H, Yuba-Kubo A, Tsukita S, Shiina N: Centriolar satellites: molecular characterization, ATP-dependent movement toward centrioles and possible involvement in ciliogenesis. J Cell Biol 1999, 147:969-980.

19. Vladar EK, Stearns T: Molecular characterization of centriole assembly in ciliated epithelial cells. J Cell Biol 2007, 178:31-42.

*20. Mori M, Hazan R, Danielian PS, Mahoney JE, Li H, Lu J, Miller ES, Zhu X, Lees JA, Cardoso WV: Cytoplasmic E2f4 forms organizing centres for initiation of centriole amplification during multiciliogenesis. Nat Commun 2017, 8:15857.

This study revealed that beyond its known function in the MCC nucleus, E2F4 also acts in the cytoplasm to promote deuterosome-mediated centriole synthesis. Doing so, it re-ignited the possibility that deuterosomes derive from PCM1-positive fibrous granules.

**21. Al Jord A, Shihavuddin A, Servignat d'Aout R, Faucourt M, Genovesio A, Karaiskou A, Sobczak-Thepot J, Spassky N, Meunier A: Calibrated mitotic oscillator drives motile ciliogenesis. Science 2017, 358:803-806.

This paper reports that key mitotic regulators including CDK1, PLK1 and APC/C are repurposed in post-mitotic mouse ependymal MCCs to control transitions between centriole 
templating, growth and disengagement phases. Strikingly, altering the length of the first phase changes the final number of centrioles synthesized, suggesting that mitotic regulators may represent hubs to scale centriole number to cell size. Beyond the strict case of MCCs, this study questions the role of cell-cycle regulators in terminal differentiation programs.

22. Vladar EK, Stratton MB, Saal ML, Salazar-De Simone G, Wang X, Wolgemuth D, Stearns T, Axelrod JD: Cyclin-dependent kinase control of motile ciliogenesis. Elife 2018, 7.

This paper demonstrates the implication of the cell-cyle kinase CDK2 in MCC centriole synthesis. It extends the findings of Al Jord et al., 2017 to an S-phase regulator, broadening the notion of repurposition of cell-cycle regulators in differentiating MCCs.

23. Gelens L, Qian J, Bollen M, Saurin AT: The Importance of Kinase-Phosphatase Integration: Lessons from Mitosis. Trends Cell Biol 2018, 28:6-21.

24. Park TJ, Mitchell BJ, Abitua PB, Kintner C, Wallingford JB: Dishevelled controls apical docking and planar polarization of basal bodies in ciliated epithelial cells. Nat Genet 2008, 40:871-879.

25. Burke MC, Li FQ, Cyge B, Arashiro T, Brechbuhl HM, Chen X, Siller SS, Weiss MA, O'Connell $C B$, Love $D$, et al.: Chibby promotes ciliary vesicle formation and basal body docking during airway cell differentiation. J Cell Biol 2014, 207:123-137.

26. Werner ME, Hwang P, Huisman F, Taborek P, Yu CC, Mitchell BJ: Actin and microtubules drive differential aspects of planar cell polarity in multiciliated cells. J Cell Biol 2011, 195:19-26.

27. Herawati E, Taniguchi D, Kanoh H, Tateishi K, Ishihara S, Tsukita S: Multiciliated cell basal bodies align in stereotypical patterns coordinated by the apical cytoskeleton. $J$ Cell Biol 2016, 214:571-586.

28. Mirzadeh Z, Han YG, Soriano-Navarro M, Garcia-Verdugo JM, Alvarez-Buylla A: Cilia organize ependymal planar polarity. J Neurosci 2010, 30:2600-2610.

29. Boutin C, Labedan P, Dimidschstein J, Richard F, Cremer H, Andre P, Yang Y, Montcouquiol M, Goffinet AM, Tissir F: A dual role for planar cell polarity genes in ciliated cells. Proc Natl Acad Sci U S A 2014, 111:E3129-3138.

*30. Mahuzier A, Shihavuddin A, Fournier C, Lansade P, Faucourt M, Menezes N, Meunier A, Garfa-Traore M, Carlier MF, Voituriez R, et al.: Ependymal cilia beating induces an actin network to protect centrioles against shear stress. Nat Commun 2018, 9:2279.

This paper reports the striking finding that ciliary beating in ependymal MCCs is needed for actin fibers to form around BBs. In turn, actin fibers help BBs to sustain cilia beating force. When actin network is impaired, beating cilia and their associated BBs may detach from the cell surface. 
31. Tateishi K, Nishida T, Inoue K, Tsukita S: Three-dimensional Organization of Layered Apical Cytoskeletal Networks Associated with Mouse Airway Tissue Development. Sci Rep 2017, 7:43783.

32. Park TJ, Haigo SL, Wallingford JB: Ciliogenesis defects in embryos lacking inturned or fuzzy function are associated with failure of planar cell polarity and Hedgehog signaling. Nat Genet 2006, 38:303-311.

33. Kunimoto K, Yamazaki Y, Nishida T, Shinohara K, Ishikawa $H$, Hasegawa T, Okanoue T, Hamada H, Noda T, Tamura A, et al.: Coordinated ciliary beating requires Odf2-mediated polarization of basal bodies via basal feet. Cell 2012, 148:189-200.

34. Antoniades I, Stylianou P, Skourides PA: Making the connection: ciliary adhesion complexes anchor basal bodies to the actin cytoskeleton. Dev Cell 2014, 28:70-80.

35. Antoniades I, Stylianou P, Christodoulou N, Skourides PA: Addressing the Functional Determinants of FAK during Ciliogenesis in Multiciliated Cells. J Biol Chem 2017, 292:488504.

36. Epting D, Slanchev K, Boehlke C, Hoff S, Loges NT, Yasunaga T, Indorf L, Nestel S, Lienkamp SS, Omran H, et al.: The Rac1 regulator ELMO controls basal body migration and docking in multiciliated cells through interaction with Ezrin. Development 2015, 142:1553.

*37. Kulkarni SS, Griffin JN, Date PP, Liem KF, Jr., Khokha MK: WDR5 Stabilizes Actin Architecture to Promote Multiciliated Cell Formation. Dev Cell 2018, 46:595-610 e593. This paper revealed the unexpected role of the chromatin modifier WDR5 in maintaining apical actin networks in MCCs. As such, it is the most recent example of proteins that are repurposed in multiple compartments of developing MCCs.

38. Bayless BA, Galati DF, Junker AD, Backer CB, Gaertig J, Pearson CG: Asymmetrically localized proteins stabilize basal bodies against ciliary beating forces. J Cell Biol 2016, 215:457-466.

39. Guirao B, Meunier A, Mortaud S, Aguilar A, Corsi JM, Strehl L, Hirota Y, Desoeuvre A, Boutin $C$, Han YG, et al.: Coupling between hydrodynamic forces and planar cell polarity orients mammalian motile cilia. Nat Cell Biol 2010, 12:341-350.

40. Mitchell B, Jacobs R, Li J, Chien S, Kintner C: A positive feedback mechanism governs the polarity and motion of motile cilia. Nature 2007, 447:97-101.

41. Walentek P, Boutin C, Kodjabachian L: Planar cell polarity in ciliated epithelia. In Cell Polarity in Development and Disease Edited by Houston D: Elsevier; 2017.

42. Mitchell B, Stubbs JL, Huisman F, Taborek P, Yu C, Kintner C: The PCP pathway instructs the planar orientation of ciliated cells in the Xenopus larval skin. Curr Biol 2009, 19:924929. 
43. Vladar EK, Bayly RD, Sangoram AM, Scott MP, Axelrod JD: Microtubules enable the planar cell polarity of airway cilia. Curr Biol 2012, 22:2203-2212.

44. Chien YH, Keller R, Kintner C, Shook DR: Mechanical strain determines the axis of planar polarity in ciliated epithelia. Curr Biol 2015, 25:2774-2784.

*45. Kim SK, Zhang S, Werner ME, Brotslaw EJ, Mitchell JW, Altabbaa MM, Mitchell BJ:

CLAMP/Spef1 regulates planar cell polarity signaling and asymmetric microtubule accumulation in the Xenopus ciliated epithelia. J Cell Biol 2018, 217:1633-1641.

This paper identifies the MT-interacting protein CLAMP as a novel regulator of PCP signalling in MCCs. CLAMP provides an important link between asymmetrically distributed membranebound PCP proteins and MT cytoskeleton, which in turn transmits polarity information to BBs of the MCC.

46. Vladar EK, Nayak JV, Milla CE, Axelrod JD: Airway epithelial homeostasis and planar cell polarity signaling depend on multiciliated cell differentiation. JCI Insight 2016, 1.

47. Khelloufi MK, Loiseau E, Jaeger M, Molinari N, Chanez P, Gras D, Viallat A:

Spatiotemporal organization of cilia drives multiscale mucus swirls in model human bronchial epithelium. Sci Rep 2018, 8:2447.

48. Azimzadeh J, Wong ML, Downhour DM, Sanchez Alvarado A, Marshall WF: Centrosome loss in the evolution of planarians. Science 2012, 335:461-463.

49. Wheeler RJ, Hyman AA: Controlling compartmentalization by non-membrane-bound organelles. Philos Trans R Soc Lond B Biol Sci 2018, 373.

*50. Huizar RL, Lee C, Boulgakov AA, Horani A, Tu F, Marcotte EM, Brody SL, Wallingford JB: A liquid-like organelle at the root of motile ciliopathy. Elife 2018, 7.

This paper reports the existence of specific particles in Xenopus epidermal MCC cytoplasm dedicated to assembly of axonemal dynein arms. Interestingly, such particles are deprived of membranes and behave as phase-separated organelles. Some of the genes mutated in Primary Ciliary Dyskinesia patients encode components of these particles that are not transferred to cilia. 